Making room for $T$ cells

\author{
Gabriel N. Maine and James J. Mulé
}

University of Michigan Medical Center, Department of Surgery, Ann Arbor, Michigan, USA

Address correspondence to: James J. Mulé, University of Michigan Medical Center, Department of Surgery, 1520C Medical Science Research Building 1, Box 0666, Ann Arbor, Michigan 48109-0666, USA. Phone: (734) 647-2779; Fax: (734) 763-4135; E-mail: jimmule@umich.edu.

J. Clin. Invest. 110:157-159 (2002). doi:10.1172/JCI200216166.

Under conditions of lymphopenia, transferred naive $T$ cells can undergo marked proliferation $(1,2)$ as a result of both $\mathrm{T}$ cell-receptor engagement and cytokine stimulation. Naive $\mathrm{T}$ cells that undergo this homeostatic proliferation also acquire characteristics of memory and effector cells as measured by phenotype, by hypersensitivity to antigen stimulation, and by increased production of IFN- $\gamma$. This phenomenon has been observed following the adoptive transfer of either naive, transgenic $T$ cells or of polyclonal, wild-type $\mathrm{T}$ cells. Recent studies using recombination-activating gene-deficient $\left(\mathrm{Rag}^{-/-}\right)$mice, CD3cdeficient mice, and irradiated normal mice as recipients have shown that memory $\mathrm{T}$ cells do not revert to naive $T$ cells under these conditions to fill the peripheral naive $T$ cell pool, as originally postulated. Rather, after lymphopenia, homeostasis-driven proliferation restores only the memory $\mathrm{T}$ cell compartment, whereas thymopoiesis is required to reconstitute the naive $T$ cell compartment $(3,4)$.

Cytokines and dendritic cells contribute to the induction and maintenance of homeostatic $\mathrm{T}$ cell proliferation. Using a coculture system without foreign antigen, Ge et al. (5) demonstrated that such proliferation requires interaction of the $T$ cell receptor with self-peptide MHCs on dendritic cells. In vitro, this response also requires dendritic cell-derived IL-15 and can be inhibited by the introduction of $\mathrm{CD} 4{ }^{+} \mathrm{CD} 25^{+}$regulatory $\mathrm{T}$ cells. In animals recovering from lymphopenia following bone marrow transplantation (BMT), dendritic cells also expedite $T$ cell reconstitution and activation $(6,7)$. Recent studies show that IL-7 and IL-15 jointly regulate homeostatic proliferation of memory phenotype $\mathrm{CD}^{+} \mathrm{T}$ cells $(8,9)$, whereas IL-7 on its own enhances naive T cell survival (10).

\section{Lymphopenia and antitumor immunity}

Studies in the late 1970s provided the first evidence that the induction of lymphopenia by sublethal total body irradiation might be beneficial for the treatment of tumors in mice (11). In one such study, irradiation performed 6-8 days after tumor cell injection, when the tumors were first palpable, led in some instances to the complete regression of subcutaneous MCA-1315 tumors in BALB/c mice. Crucially, this total body irradiation did not affect the tumor itself; rather,

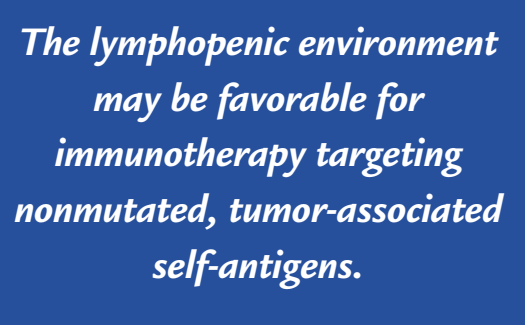

tumor suppression occurred because of the removal of host lymphoid cells. In related studies using an MCA-1425 sarcoma, sublethal irradiation was shown to produce a lesser, transient effect, apparently correlating with its lower level of inherent tumor immunogenicity in vivo.

Induction of lymphopenia has also been found to be critical for the specific localization (homing) of radiolabeled immune long-lived small lymphocytes (LLSL) to syngeneic tumors (12). Adoptive transfer of immune LLSL into tumor-bearing mice led to selective localization, but only when host animals were subjected to sublethal levels of total body irradiation prior to the introduction of these cells. Because the dose of radiation used in these experiments depleted cells in the lymphoid compartments, recirculating lymphocytes, nonspecifically localizing in tumors, were originally proposed to exit from these tumors and to help reconstitute the lymphocytes killed by irradiation, thereby increasing the relative proportion of lymphocytes specific for tumor-specific transplantation antigens in the respective tumors (12). However, the implication of these early findings has become clear only recently, nearly 25 years later.

\section{Defining the preconditions for antitumor immunity}

In this issue of the JCI, Dummer and colleagues (13) tested whether adoptive transfer of a polyclonal population of lymph node cells into lymphopenic animals could confer anti-tumor immunity by eliciting the proliferation of $\mathrm{T}$ cells specific for nonmutated self-antigens on tumor cells. They observed that B78D14 melanoma growth could be suppressed by adoptive transfer of lymph node cells into sublethally-irradiated mice and could also be modestly inhibited by irradiation alone, possibly due to homeostatic proliferation of host-derived $\mathrm{T}$ cells. This anti-tumor response depended on the transfer of a polyclonal population of $\mathrm{CD} 8^{+} \mathrm{T}$ cells, as mice irradiated and transfused with a $T$ cell clone specific for a non-tumor associated antigen proliferated but failed to promote anti-tumor immunity. The cells that mediated the antitumor immune response manifested cytolytic activity and secreted IFN- $\gamma$ in vitro when stimulated briefly with the relevant melanoma.

Dummer et al. (13) also found that $\mathrm{CD}^{+}$and $\mathrm{CD}^{+} \mathrm{T}$ cells from the transferred inoculum could undergo extensive proliferation in secondary lymphoid tissues. This proliferation, they argue, is essential for infiltration of effector cells into the tumor site. Thus, adoptive transfer of wild-type lymph node cells into irradiated lymphotox- 
in- $\alpha$-deficient mice, which lack lymph nodes and show perturbed architecture of splenic white pulp, led to homeostatic proliferation in the spleen but failed to confer anti-tumor immunity. Similarly, irradiated wildtype mice enjoyed no protection from tumor growth when they were transfused with lymph node cells deficient in $\beta 7$ integrin and CD62L - adhesive molecules that are required for entry into peripheral lymph nodes.

The present finding that donorderived $\mathrm{T}$ cells in transfused animals infiltrate extensively into the tumor tissue (13) extends an earlier observation (12) that adoptively transferred, radiolabeled lymphocytes localize specifically to tumors following the induction of lymphopenia. At first glance, however, the data appear at odds with those reported in the late 1970s by Hellstrom et al. (11). In the latter study, the inhibition of tumor growth by total body irradiation of mice carrying small tumors could be blocked (indeed, tumor growth was actually facilitated in some instances) by the adoptive transfer of naive syngeneic spleen $\mathrm{T}$ cells. The discrepancy in the outcome of these two studies may not be related to any differences in the tumor types employed, but rather to the source of $\mathrm{T}$ cells transferred. The injection of relatively large numbers of whole spleen cells has been shown to tolerize virgin T cells in vivo $(14,15)$, apparently because splenic preparations contain large numbers of $\mathrm{T}$ and $\mathrm{B}$ cells (which are unable to costimulate the host's virgin $T$ cells) but include very few professional antigen-presenting cells. While dendritic cells can prime newborn $\mathrm{T}$ cells to antigens and thus prevent the induction of neonatal tolerance (15), the lymph node preparations used by Dummer et al. (13), like the splenic preparations of Hellstrom et al. (11), employed T cell-enriched populations. Hence, neither donor B cells nor dendritic cells should have contributed to tumor responses in these studies. Another potentially important cell type, which could well have been present in one or the other of these inocula, is the $\mathrm{CD} 4^{+} \mathrm{CD} 25^{+}$regulatory $\mathrm{T}$ cell. Such cells potently inhibit the proliferation of naive $T$ cells cocultured with dendritic cells (5). The different antitumor effects observed in the recipient, lymphopenic tumor-bearing mice might therefore be explained by the presence of this inhibitory cell type in the spleen but not in the lymph node. Indeed, Dummer et al. found that depletion of these regulatory cells in the lymph node-derived inocula did not influence the outcome of the cell transfer; as might be expected if this cell subset is poorly represented, tumor inhibition remained incomplete in either the presence or absence of $\mathrm{CD}^{+} \mathrm{CD}^{2} 5^{+}$regulatory $\mathrm{T}$ cells. The hypothesis that the spleen contains more such cells - and that they limit the ability of splenic $\mathrm{T}$ cell preparations to undergo optimal homeostatic expansion and suppress host tumors - remains to be tested.

The current study also raises an issue about the mechanisms of homeostasisdriven $\mathrm{T}$ cell proliferation during lymphopenia. Despite the common assertion that simply making space (the so-called "Lebensraum effect") induces such $\mathrm{T}$ cell proliferation, the selective depletion or elimination of $\mathrm{CD}^{+}$ $\mathrm{CD}_{25}{ }^{+}$regulatory $\mathrm{T}$ cells following total body irradiation may also contribute to homeostatic proliferation by removing an impediment to naive $\mathrm{T}$ cell proliferation. Evidence for an inhibitory effect of $\mathrm{CD}^{+} \mathrm{CD} 25^{+}$cells comes not just from the cell coculture work described above (5), but also from in vivo observations indicating that these cells inhibit autologous $\mathrm{T}$ cell proliferation in human lung cancer patients (16) and that their selective removal can enhance tumor vaccine efficacy in mice (17). The negative findings in the present study (that CD4 $\mathrm{CD} 25^{+}$cell depletion from lymph node preparations has no effect on $T$ cell-dependent tumor suppression; ref. 13) does not exclude a role for these regulatory cells, but suggests that the number of donor $\mathrm{CD} 4^{+} \mathrm{CD} 25^{+}$cells in these preparations is relatively low. Most likely, both the depletion of endogenous host $\mathrm{CD} 4^{+} \mathrm{CD} 25^{+}$cells and the appearance of more physical space for newly proliferating $\mathrm{T}$ cells are key to homeostatic expansion of T cells in lymphopenic animals, and thus to the successful suppression of tumors by adoptively transferred naive $T$ cells.

\section{Lymphopenia, homeostasis-driven T cell proliferation, and tumor vaccines}

Inducing a lymphopenic state with subsequent induction of homeostasis- driven $\mathrm{T}$ cell proliferation offers several potential advantages for active vaccination against tumors. First, sublethal total body irradiation or other regimens to induce lymphopenia may in some cases reduce or eliminate tumor-induced immune suppression (18). Second, lymphomyeloid reconstitution of either donor or host origin may overcome inherent defects in $\mathrm{T}$ cell signaling, processing, or presentation, and may strengthen the costimulatory functions of antigen-presenting cells (19). Third, immunization following the induction of lymphopenia may serve to educate the developing $\mathrm{T}$ cell repertoire to tumor antigens and thus may be more efficacious in this environment (15).

There is now mounting evidence in several settings that dendritic celldirected responses to antigens are particularly efficient at early stages of $\mathrm{T}$ cell reconstitution following lymphopenia. Thus, as the authors of the current study point out, an antiviral effect observed by Oehen and Brduscha-Riem (20) in homeostatically-expanding lymphocytic choriomeningitis virus-specific transgenic $\mathrm{T}$ cells depends on the presence of the antigen at the early stages of the expansion. Another study (21) evaluating the effects of transplanted bone marrow cells on the host immune system during early lymphoid reconstitution following lethal total body irradiation showed that dendritic cells pulsed with whole tumor lysates can prime specific and long-lasting antitumor immune responses under these conditions, allowing the recipient animal to reject a challenge with a syngeneic breast tumor or to reduce the growth of established tumors. Spleen T cells obtained from mice immunized with tumor lysate-pulsed dendritic cells after bone marrow transfer showed increased production of tumor-specific IFN- $\gamma$, as was also seen in the current study (13). Interestingly, although the systemic administration of recombinant IL-7 might have been expected to expedite lymphoid reconstitution following BMT, we have found that this treatment actually eliminates successful immunization (W. Asavaroengchai and J.J. Mulé, unpublished results), suggesting that maintenance of the lymphopenic state during antigen presentation is required for generating antitumor immunity by the dendritic 
cell-based vaccine; hastening immune recovery therefore appears to be counterproductive. Likewise, recent related work by Bacci et al. (6) shows that injection of dendritic cells pulsed with yeast RNA as early as one day after lethal total body irradiation and allogeneic BMT efficiently promotes antifungal protective immunity. Of interest, the dendritic cell immunizations were shown to both increase the number and accelerate the functional recovery of Candida-specific IFN- $\gamma$ donor $\mathrm{T}$ cells early on during recovery from lymphopenia.

Finally, Hu et al. (22) have now reported that tumor-specific $\mathrm{T}$ cells can preferentially expand in tumor vaccine-draining lymph nodes following a melanoma vaccine. To model the effects of immune reconstitution without need for irradiation of the host, these authors used RAG1-deficient mice that were transfused with naive $\mathrm{T}$ cells from normal mice. They found that both tumor specific Tc1 and Th1 cells increased dramatically in vaccinated, reconstituting RAG1 hosts compared to controls. Functionally, the $\mathrm{T}$ cells exhibited a higher level of melanoma-specific cytotoxicity in vitro and were significantly more potent at mediating tumor regression upon adoptive transfer into recipients bearing lung metastases than were cells from similarly immunized but fully immunocompetent animals. Significantly, vaccination was best performed concomitantly with reconstitution, as delayed vaccination resulted in T cells with less antitumor potency as lymphopenia waned.

Consistent with this growing body of work, the current study by Dummer and colleagues (13) demonstrates that the lymphopenic environment may be favorable for immunotherapy targeting nonmutated, tumor-associated self-antigens. To date, studies of home- ostasis-driven $\mathrm{T}$ cell proliferation have been limited to the mouse. Whether or not a similar phenomenon can be reproduced in humans remains to be determined. One hurdle, of course, will be how best, and by what means, to measure homeostasis-driven $\mathrm{T}$ cell proliferation in lymphopenic patients. Another will be to understand the timing of this event and to determine whether immunization at a critical point in homeostasis-driven $\mathrm{T}$ cell proliferation is necessary to achieve effective or superior immune priming in vivo. Given the exciting findings in animal tumor models, clinical trials to test vaccine efficacy in the setting of lymphopenia and homeostasis-driven $T$ cell proliferation in cancer patients seem warranted.

\section{Acknowledgments}

We thank Bernard Fox and his colleagues for allowing us to cite their work prior to its publication.

1. Cho, B.K., Rao, V.P., Ge, Q., Eisen, H.N., and Chen, J. 2000. Homeostasis-stimulated proliferation drives naive $T$ cells to differentiate directly into memory T cells. J. Exp. Med. 192:549-556.

2. Goldrath, A.W., Bogatzki, L.Y., and Bevan, M.J. 2000. Naive T cells transiently acquire a memory-like phenotype during homeostasis-driven proliferation. J. Exp. Med. 192:557-564.

3. Ge, Q., Hu, H., Eisen, H.N., and Chen, J. 2002. Different contributions of thymopoiesis and homeostasis-driven proliferation to the reconstitution of naive and memory T cell compartments. Proc. Natl. Acad. Sci. USA. 99:2989-2994.

4. Tanchot, C., et. al. 2002. Conversion of naïve T cells to a memory-like phenotype in lymphopenic hosts is not related to a homeostatic mechanism that fills the peripheral naive $\mathrm{T}$ cell pool. J. Immunol. 168:5042-5046.

5. Ge, Q., Palliser, D., Eisen, H.N., and Chen, J. 2002. Homeostatic $\mathrm{T}$ cell proliferation in a $\mathrm{T}$ cell-dendritic cell coculture system. Proc. Natl. Acad. Sci. USA. 99:2983-2988.

6. Bacci, A., et al. 2002. Dendritic cells pulsed with fungal RNA induce protective immunity to Candida albicans in hematopoietic transplantation. J. Immunol. 168:2904-2913.

7. Zhang, Y., et. al. 2002. Preterminal host dendritic cells in irradiated mice prime $\mathrm{CD}^{+} \mathrm{T}$ cell-mediated acute graft-versus-host disease. J. Clin. Invest 109:1335-1344. doi:10.1172/JCI200214989.

8. Prlic, M., Lefrancois, L., and Jameson, S.C. 2002.
Multiple choices: regulation of memory CD8 T cell generation and homeostasis by interleukin (IL)-7 and IL-15. J. Exp. Med. 195:F49-F52.

9. Tan, J.T., Ernst, B., Kieper, W.C., LeRoy, E., Sprent, J., and Surh, C.D. 2002. Interleukin (IL)-15 and IL-7 jointly regulate homeostatic proliferation of memory phenotype $\mathrm{CD} 8^{+}$cells but are not required for memory phenotype CD4 ${ }^{+}$cells. J. Exp. Med. 145:1523-1532.

10. Rathmell, J.C., Farkash, E.A., Gao, W., and Thompson, C.B. 2002. IL-7 enhances the survival and maintains the size of naive $\mathrm{T}$ cells. J. Immunol. 167:6869-6876.

11. Hellstrom, K.E., Hellstrom, I., Kant, J.A., and Tamerius, J.D. 1978. Regression and inhibition of sarcoma growth by interference with a radiosensitive T-cell population. J. Exp. Med. 148:799-804.

12. Mulé, J.J., Jones, F.R., Hellstrom, I., and Hellstrom, K.E. 1979. Selective localization of radiolabeled immune lymphocytes into syngeneic tumors. J. Immunol. 123:600-606.

13. Dummer, W., et al. 2002. T cell homeostatic proliferation elicits effective antitumor autoimmunity. J. Clin. Invest. 110:185-192. doi:10.1172/JCI200215175.

14. Fuchs, E.J., and Matzinger, P. 1992. B cells turn off virgin but not memory $\mathrm{T}$ cells. Science. 258:1156-1159.

15. Ridge, J.P., Fuchs, E.J., and Matzinger, P. 1996. Neonatal tolerance revisited: turning on newborn $\mathrm{T}$ cells with dendritic cells. Science. 271:1723-1726.

16. Woo, E.Y., et al. 2002. Cutting edge: regulatory T cells from lung cancer patients directly inhibit autologous $\mathrm{T}$ cell proliferation. J. Immunol. 168:4272-4276.

17. Sutmuller, R.P., et al. 2001. Synergism of cytotoxic $\mathrm{T}$ lymphocyte-associated antigen 4 blockade and depletion of $\mathrm{CD} 25\left(^{+}\right)$regulatory $\mathrm{T}$ cells in antitumor therapy reveals alternative pathways for suppression of autoreactive cytotoxic T lymphocyte responses. J. Exp. Med. 194:823-832.

18. Sondak, V., Magner, P.D., Shu, S., and Chang, A.E. 1991. Suppressive effects of visceral tumor on the generation of antitumor T cells for adoptive immunotherapy. Arch. Surg. 126:442-446.

19. Finke, J.H., Zea, A.H., Stanley, J., Longo, D.L., and Ochoa, A.C. 1993. Loss of T-cell receptor zeta chain and p53lck in T-cells infiltrating human renal cell carcinoma. Cancer Res. 53:5613-5616.

20. Oehen, S., and Brduscha-Riem, K. 1999. Naive cytotoxic T lymphocytes spontaneously acquire effector function in lymphocytopenic recipients: a pitfall for $\mathrm{T}$ cell memory studies? Eur. J. Immunol. 29:608-614.

21. Asavaroengchai, W., Kotera, Y., and Mulé, J.J. 2002. Tumor lysate-pulsed dendritic cells can elicit an effective antitumor immune response during early lymphoid recovery. Proc. Natl. Acad. Sci. USA. 99:931-936

22. Hu, H.-M., Poehlein, C.H., Urba, W.J., and Fox, B.A. 2002. Advances in brief: development of antitumor immune responses in reconstituted lymphopenic hosts. Cancer Res. In press. 